\title{
Functional analysis of Cti6 core domain responsible for recruitment of epigenetic regulators Sin3, Cyc8 and Tup1
}

\author{
Rasha Aref ${ }^{1,2}$ D $\cdot$ Hans-Joachim Schüller ${ }^{2}$
}

Received: 10 August 2020 / Revised: 10 September 2020 / Accepted: 15 September 2020 / Published online: 26 September 2020

(c) The Author(s) 2020

\begin{abstract}
Mapping of effective protein domains is a demanding stride to disclose the functional relationship between regulatory complexes. Domain analysis of protein interactions is requisite for understanding the pleiotropic responses of the respective partners. Cti6 is a multifunctional regulator for which we could show recruitment of co-repressors Sin3, Cyc8 and Tup1. However, the responsible core domain tethering Cti6 to these co-repressors is poorly understood. Here, we report the pivotal domain of Cti6 that is indispensable for co-repressor recruitment. We substantiated that amino acids 450-506 of Cti6 bind PAH2 of Sin3. To analyse this Cti6-Sin3 Interaction Domain (CSID) in more detail, selected amino acids within CSID were replaced by alanine. It is revealed that hydrophobic amino acids V467, L481 and L491 L492 L493 are important for Cti6Sin 3 binding. In addition to PAH2 of Sin3, CSID also binds to tetratricopeptide repeats (TPR) of Cyc8. Indeed, we could demonstrate Cti6 recruitment to promoters of genes, such as $R N R 3$ and $S M F 3$, containing iron-responsive elements (IRE). Importantly, Sin 3 is also recruited to these promoters but only in the presence of functional Cti6. Our findings provide novel insights toward the critical interaction domain in the co-regulator Cti6, which is a component of regulatory complexes that are closely related to chromatin architecture and the epigenetic status of genes that are regulated by pleiotropic co-repressors.
\end{abstract}

Keywords Histone deacetylase Rpd $3 \cdot$ Corepressor Sin $3 \cdot$ Cti6 $\cdot$ Protein-protein interaction

\section{Introduction}

The functional associations and affinities that take place between proteins are at the essence of numerous regulatory processes and their structural principle helps to provide context in molecular function. Identifying domains within regulatory protein complexes are a prerequisite step for devising protein structure and functional annotation. Transcriptional activity of genes is governed by regulatory complexes that

Communicated by M. Kupiec.

Electronic supplementary material The online version of this article (https://doi.org/10.1007/s00294-020-01109-4) contains supplementary material, which is available to authorized users.

Rasha Aref

rasha_aref@agr.asu.edu.eg

1 Department of Genetics, Faculty of Agriculture, Ain Shams University, Shoubra El-Khaymah, Cairo 11241, Egypt

2 Center for Functional Genomics of Microbes, Abteilung Molekulare Genetik Und Infektionsbiologie, Felix-Hausdorff-Straße 8, 17487 Greifswald, Germany assemble/disassemble on the promoter region and control the chromatin architecture (Lemon and Tjian 2000; Cairns 2009). In eukaryotic systems, Rpd3 histone deacetylase addresses defined control regions in association with different co-repressor complexes (Kadosh and Struhl 1998). In yeast, one of the associated subunits of the Rpd3L histone deacetylase complex is the regulatory protein Cti6, implicated in many regulatory pathways (Papamichos-Chronakis et al. 2002; Puig et al. 2004). In most if not all targeted cellular functions, Cti6 fulfills the respective function associated with transcriptional regulatory complexes. Among these regulatory complexes that play pivotal roles in yeast cell biology are Cyc8-Tup1 (Váchová and Palková 2019) and Sin3 (Adams et al. 2018) complexes. Both co-repressors include but are not limited to the following features: (1) contain domains for multiple protein interactions via paired amphipathic helices (PAH) and tetratricopeptide repeat (TPR) motifs in Sin3 and Cyc8, respectively (Wang et al. 1990; Tzamarias and Struhl 1994); (2) do not encode DNA-binding proteins but are rather targeted to selected promoters through regulatory proteins (Kliewe et al. 2020; Davie et al. 2003) which in turn regulates transcription; 
(3) implicated in numerous regulatory pathways (Maqani et al. 2018; Chaubal and Pile 2018), thus conferring pleiotropic properties to these co-repressors; (4) could perform dual sides of gene transcription control either positively or negatively by configuring chromatin architecture based on its histone deacetylase activity (Adams et al. 2018; Davie et al. 2003; Papamichos-Chronakis et al. 2002). Strikingly, Cti6 (Cyc8-Tup1 Interacting Protein 6) interacts with $\operatorname{Sin} 3$ and Cyc8 co-repressor complexes independently (Papamichos-Chronakis et al. 2002; Puig et al. 2004). Cti6 has been initially identified as a positive regulator as it interacts with SAGA coactivator complex and thence activates transcription of target genes (Papamichos-Chronakis et al. 2002). Subsequent studies showed that Cti6 is a subunit of the Rpd3L histone deacetylase complex (Puig et al. 2004; Carrozza et al. 2005), which indicates that Cti6 acts as a repressor as well. Another striking feature of Cti6 is that it can bind more than one regulatory protein altogether, such as Cyc8/Tup1 and SAGA complex (Papamichos-Chronakis et al. 2002). Various studies reported the involvement of Cti6 in numerous physiological pathways, such as transcriptional de-repression of GAL1 and $A N B 1$, which respond to diverse signals (low glucose and oxygen, respectively). In addition, Cti6 is important for iron metabolism in yeast (Puig et al. 2004). Cti6 has a differential role for certain iron-regulated genes, such as ARN1, FET3, SMF3 and RNR3 (Kaplan et al. 2006; Puig et al. 2004). Interestingly, all detected Cti6 regulatory functions have been fulfilled through association either with $\operatorname{Sin} 3 / \mathrm{Rpd} 3$ or Cyc8/Tup1 co-repressor complexes (Papamichos-Chronakis et al. 2002; Puig et al. 2004). Although Cti6 mediates interaction with many regulatory complexes, the pivotal domain of Cti6 that leads to the recruitment of $\operatorname{Sin} 3$ or Cyc8 co-repressors has not yet been investigated. In addition, no analysis has been carried out so far regarding the required characteristics of such a domain to ensure Cti6 function in the respective cellular pathways.

Therefore, it was worthy to extend the scope on the regulatory protein $\mathrm{Cti} 6$ by characterizing extensively its interaction with both co-repressors and explore additional aspects regarding particular Cti6 target genes. In this report, we show that Cti6 not only interacts directly with both Sin3 and Cyc8/Tup1 co-repressors but also identify precisely the minimal interaction domain responsible for binding of all tested co-repressors. Our in vitro interaction studies showed that amino acids $450-506$ of Cti6 bind PAH 2 of Sin 3 and the same domain mediates the interaction with Cyc8 corepressor as well. To analyse Cti6-Sin3 interaction domain (CSID) in more detail, selected amino acids within CSID were replaced by alanine. It turned out that hydrophobic amino acids V467, L481 and at least one of the lysines at the positions 491-493 is important for Cti6-Sin3 binding. The results of this work also suggest that repression is not executed entirely via Sin3, but rather CSID is also important for contacting pleiotropic co-repressor Cyc8 via its tetratricopeptide repeats (TPR). Chromatin immunoprecipitation (ChIP) analyses demonstrated Cti6 recruitment to promoters of genes, such as $R N R 3$ and $S M F 3$, containing iron-responsive elements (IRE). Importantly, Sin3 was also recruited to these promoters but only in the presence of functional Cti6.

\section{Materials and methods}

\section{Yeast strains and media}

According to the various experimental purposes, various strains of the yeast Saccharomyces cerevisiae were used. All of the following strains are haploid. S. cerevisiae strain C13-ABY.S86 lacking four vacuolar proteinases ( $\mathrm{pral}$ prb1 prcl cps 1; De Antoni and Gallwitz 2000) was used for protein-protein interaction assays. Strains used for ChIP analyses were derived from C13-ABY.S86 by gene replacement experiments (construction of epitope-tagged variant of CTI6 at its authentic chromosomal positions; see below) or gene disruptions (introduction of deletion mutant allele for $c t i 6$ ). To obtain such isogenic null mutant, disruption plasmid pRAR81 ( $\triangle C T I 6:: L E U 2)$ was used. Complete genotypes of all strains used are available as supporting online Table 1 (section a). For ChIP analysis, the investigated strains were cultivated under respective repression conditions as described in Puig et al. (2004).

\section{In vitro interaction assays (GST pull-down)}

GST- and HA-tagged proteins used for interaction assays by affinity chromatography were synthesized by $E$. coli strain BL21 (Stratagene/Agilent). The tac promoter controlling GST fusion genes was induced with $1 \mathrm{mM}$ IPTG. Similarly, tetR-dependent gene expression was activated by $0.2 \mathrm{mg} / \mathrm{l}$ anhydrotetracycline. Derepression of MET25-dependent gene fusions was achieved by cultivating yeast transformants in the absence of methionine. GST fusion proteins synthesized in E. coli were released by sonication, immobilized on glutathione (GSH) Sepharose and subsequently incubated with yeast or bacterial total protein extracts containing HA fusions. To avoid unspecific interactions, protein extracts were pre-cleared by treatment with GSH Sepharose beads prior to incubation with GST fusions. Details on washing steps at intermediary stringency have been described (Wagner et al. 2001). After release of GST fusions with free GSH $(10 \mathrm{mM})$, eluates were separated by SDS/PAGE and proteins transferred to a filter. Following incubation with anti-HAperoxidase conjugate, HA fusion proteins were detected with POD chemiluminescent substrate (antibody conjugate and substrate from Roche Biochemicals). 


\section{Two-hybrid assays}

To perform two-hybrid assays, strain PJ69-4A was used

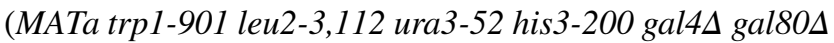

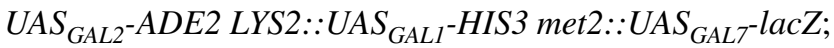
James et al. 1996). DNA fragments encoding interaction domains of Sin3 (PAH1 \& PAH2) were inserted into plasmids pGBD-C1 $\left(2 \mu \mathrm{m}\right.$ GAL4 $4_{\text {DBD }}$ TRPI) while Cti6 domains (aa 351-506; aa 430-506; aa 450-506) were inserted in pGAD-C1 $\left(2 \mu \mathrm{m} \mathrm{GAL4}\right.$ TAD $_{\text {LEU2). Double-transformed }}$ strains containing both types of fusion plasmids were first selected on medium lacking leucine and tryptophan (-L-T) and subsequently transferred to medium devoid of adenine (-L-T-A).

\section{Site-directed mutagenesis}

To alter selected residues in the coding region of Cti6, the QuikChange site-directed mutagenesis kit of Stratagene was used. To obtain mutations within CSID, plasmid pRAR47 containing the Cti6 coding region was used. To replace selected residues against alanine, we used pairs of mutagenic primers introducing a GCA codon instead of the natural codon, flanked by $15-19$ nucleotides on both the sides. DNA sequencing was used to confirm the presence of the desired mutant alleles of cti6 (V467A, L481A and L491A L492A L493A) and the absence of any other change in the plasmids obtained (pRAR20, pRAR37, pRAR49, pRAR65-67).

\section{Chromatin immunoprecipitation}

Essentially, chromatin immunoprecipitation (ChIP) analysis followed the procedure described by Cobb and van Attikum (2010). Chromosomal locus CTI6 was modified such that it expressed a His-tagged Cti6 without alteration of gene copy number or control region. Tagging was performed by transformation of strain C13-ABY.S86 with a gene-specific modification fragment and selection for resistance against geneticin. The modification fragment was amplified by PCR, using gene-specific primers and plasmid pU6H3HA as a template (contains a His6-HA3kanMX cassette; De Antoni and Gallwitz 2000). A strain which encodes epitope-tagged Sin3 (FKY11) was kindly provided by F. Kliewe; a CTI6 gene deletion was introduced into FKY11 strain. The resulting strains RAY1 (CTI6-HIS CHA $_{3}$-kanMX), FKH11 (SIN3-HIS -HA $_{3}$ kanMX) and its isogenic cti6 derivative RAY3 ( $\Delta$ cti6 SIN3-HIS $S_{6}-H A_{3}$-kanMX) grew until mid-log phase and were treated with formaldehyde for $15 \mathrm{~min}$. The crosslinking reaction was subsequently quenched for $5 \mathrm{~min}$ by addition of glycine to a final concentration of $125 \mathrm{mM}$. After lysis, cells were sonicated five times for $30 \mathrm{~s}$ to shear chromatin, using a Bandelin Sonoplus UW 70 microtip
(35\% power). After sonication, lysates were centrifuged for $10 \mathrm{~min}$ at $16,000 \mathrm{~g}$ to remove insoluble material and incubated for at least $4 \mathrm{~h}$ with His-Tag Dynabeads ${ }^{\circledR}$ (Invitrogen/Dynal $\left.{ }^{\circledR}\right)$. After elution of affinity-purified proteins and bound DNA with a buffer containing $300 \mathrm{mM}$ imidazole, cross-linking was reversed by heating to $65{ }^{\circ} \mathrm{C}$ overnight. DNA was recovered and analyzed by PCR (29 amplification cycles), using specific primers against promoters of RNR3 (- 400/- 100) and SMF3 (-350/- 80) or $A C T 1$ gene $(+841 /+1165)$ as a control.

\section{Plasmid constructions}

To perform interaction assays, Escherichia coli expression plasmids (derived from pGEX-2TK; GE Healthcare) encoding various glutathione S-transferase (GST) fusions were constructed. Length variants of coding regions of CTI6 gene were amplified by PCR and fused behind GST. Similarly, HA-tagged length variants of Sin3 representing PAH domains, Cyc8 and Tup1 were expressed in yeast using plasmid p426-MET25HA (Mumberg et al. 1994). For bacterial expression of selected Sin3 variants, plasmid pASKIBA5 (tetR-regulated; IBA, Göttingen, Germany) was used. Yeast expression plasmid pCW117 used for synthesis of $\mathrm{HA}_{3}$-tagged Sin3 (full-length) has been described (Wagner et al. 2001). For bacterial synthesis of epitope-tagged Sin3, Cyc8 and Tup1 plasmids pSW11 ( $\mathrm{HA}_{3}$-SIN3; full-length), pFK77 ( $\mathrm{HA}_{3}$-CYC8; encoding aa 1-398 representing the TPR-containing domain) and pRAR110 ( $\mathrm{HA}_{3}-T U P 1$; fulllength) derived from pASK-IBA5 (tetR-dependent; IBA, Göttingen, Germany) were used. To confirm authenticity of gene fragments obtained by PCR, GST fusions which encode minimal length variants of Cti6 were verified by DNA sequencing (LGC Genomics, Berlin, Germany). Plasmid names and fused sequences are mentioned in legends of figures and are described in detail in supporting online Table 1 (section b). Gene-specific primers used for PCR amplifications are available as supporting online Table 1 (section c). Plasmid pRAR81 was constructed by established procedures to disrupt the CTI6 gene ( $\triangle c t i 6:: L E U 2)$. To construct this plasmid, flanking sequences upstream and downstream of the respective coding regions were amplified by PCR and inserted on both sides of the selection marker, allowing total deletion of the CTI6 reading frame.

\section{Miscellaneous procedures}

Transformation of $S$. cerevisiae strains, selection for yeast transformants on the respective synthetic media, PCR amplification and $\beta$-galactosidase assays have been described (Schwank et al. 1995; Wagner et al. 2001). 


\section{Results}

\section{Cti6 interacts directly with the pleiotropic co-repressor $\operatorname{Sin} 3$}

Large-scale approaches, such as tandem affinity purification, have been used to analyze multiprotein complexes in S. cerevisiae (Gavin et al. 2002; Ho et al. 2002). Interestingly, Cti6 protein was independently identified when Rpd 3 and Sin 3 were affinity-purified using the tandem affinity purification epitope. Both Rpd 3 and Sin 3 copurified with Cti6 when the Ume1-Flag transcription factor was immunoprecipitated with anti-FLAG antibody (Ho et al. 2002).

Puig et al. (2004) have proved the functional relationship between Cti6 and Sin3, which suggests that these proteins might interact with one another. In support of this hypothesis, we used affinity chromatography as a suitable technique for demonstrating interaction in vitro. A glutathione S-transferase (GST)-Cti6 fusion protein (amino acids 1-506, comprising full length) was synthesized in Escherichia coli and subsequently bound to glutathione (GSH) Sepharose. A protein extract from S. cerevisiae (HA-Sin 3 full length) was added to this affinity matrix. After intensive washing with increasing stringency, specifically bound protein was eluted by the addition of free GSH.

As is shown in Fig. 1a by immunodetection with anti$\mathrm{HA}$-antibody, the $175 \mathrm{kDa}$ protein $\mathrm{HA}_{3}$-Sin 3 could be bound by GST-Cti6 under stringent conditions but not by GST. Thus, Cti6 may execute its function by recruiting the general co-repressor Sin3. As interaction experiments performed with protein extracts from yeast cannot completely rule out indirect interactions mediated by distinct factors, $\mathrm{HA}_{3}$-Sin 3 was thereafter also synthesized in E. coli. Since bacterial protein extracts should not contain yeast-specific factors, a direct interaction can be concluded by the use of $\mathrm{HA}_{3}$-Sin 3 from E. coli. As is apparent from Fig. 1b, identical results were indeed obtained with extracts from E. coli, indicating that interaction between Cti6 and Sin3 occurs directly.

\section{PAH1 and PAH2 of Sin3 contact Cti6 in vitro}

We, thus, wished to establish a physical map of interacting domains within Sin3 and Cti6. Sin3 contains four PAH motifs, which have been proposed to mediate various protein-protein interactions (Wang and Stillman 1993). Therefore, a GST-Cti6 fusion immobilized on GSH sepharose was incubated with HA-Sin3 length variants from yeast, representing individual structural and functional (a) Input

Pull- down

\begin{tabular}{cc}
\hline $\begin{array}{c}\text { HA fusion in } \\
\begin{array}{c}\text { S. cerevisiae } \\
\text { extract }\end{array}\end{array}$ & $\begin{array}{c}1-1536 \\
\text { aa }\end{array}$ \\
\hline $\mathrm{HA}_{3}-\mathrm{Sin} 3$ & \\
\hline
\end{tabular}

(b) Input

\begin{tabular}{cc}
\hline $\begin{array}{c}\text { HA fusion in } \\
\text { E. coli } \\
\text { extract }\end{array}$ & $\begin{array}{c}\text { aa } \\
\text { an }\end{array}$ \\
\hline $\mathrm{HA}_{3}-\mathrm{Sin} 3$ & \\
\hline
\end{tabular}

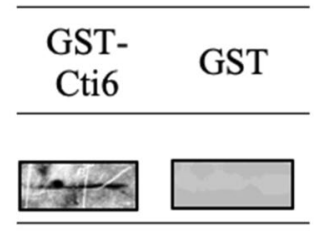

Pull- down

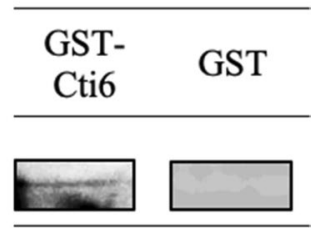

Fig. 1 In vitro interaction of Cti6 with $\operatorname{Sin} 3$ shown by affinity chromatography. a Full length of Cti6 was fused with GST, immobilized on GSH Sepharose and incubated with protein extract from yeast transformants containing full-length $\mathrm{HA}_{3}$-Sin 3 synthesized by S. cerevisiae (strain C13-ABY.S86, plasmid pCW117). b Bacterially synthesized $\mathrm{HA}_{3}$-Sin3 (E.coli strain BL21, plasmid pSW11) was incubated with GST-Cti6 (full-length fusion protein) bound to GSH Sepharose. GST-Cti6 fusion is encoded by plasmid pRAR3 (aa 1-506), GST vector was used as a negative control. Extracts containing $75 \mu \mathrm{g}$ of total protein were analyzed for the input control. To achieve comparable amounts of HA-Sin3 for the interaction assay, total protein was adjusted accordingly

domains (PAH1-PAH4, HID). As can be seen in Fig. 2, amino acids $1-300$ and $301-600$ of $\operatorname{Sin} 3$ comprising its domains PAH1 and PAH2, respectively, are sufficient for interaction with Cti6. No interaction was detected with constructs representing C-terminal sequences of $\operatorname{Sin} 3$.

\section{CSID identified as the Cti6-Sin3 interaction domain}

Vice versa, we also mapped Cti6 domains required for Sin3 interaction. GST fusions of Cti6 length variants were immobilized and subsequently incubated with HA-tagged Sin3, synthesized in S. cerevisiae. As is apparent from Fig. 3a, the plant homeodomain (PHD) of Cti6 was dispensable for its interaction with Sin3. Instead, a domain of 56 amino acids in the C-terminus turned out as the CSID (residues 450-506), which is able to interact with PAH1 and PAH2 of Sin3.

To confirm our in vitro results obtained by GST pull-down, we used two hybrid analyses as a suitable technique for demonstrating interaction in vivo. Length variants of $\operatorname{Sin} 3$ comprising PAH1 (aa 1-300) and PAH2 (aa 301-888) were fused with the DNA-binding domain (DBD) of Gal4. Binding domains of Cti6 which have been shown to bind $\operatorname{Sin} 3$ in vitro were fused with Gal4 transcriptional-activation domain (TAD). Sin3-Cti6 interactions in vivo should reconstitute a functional 


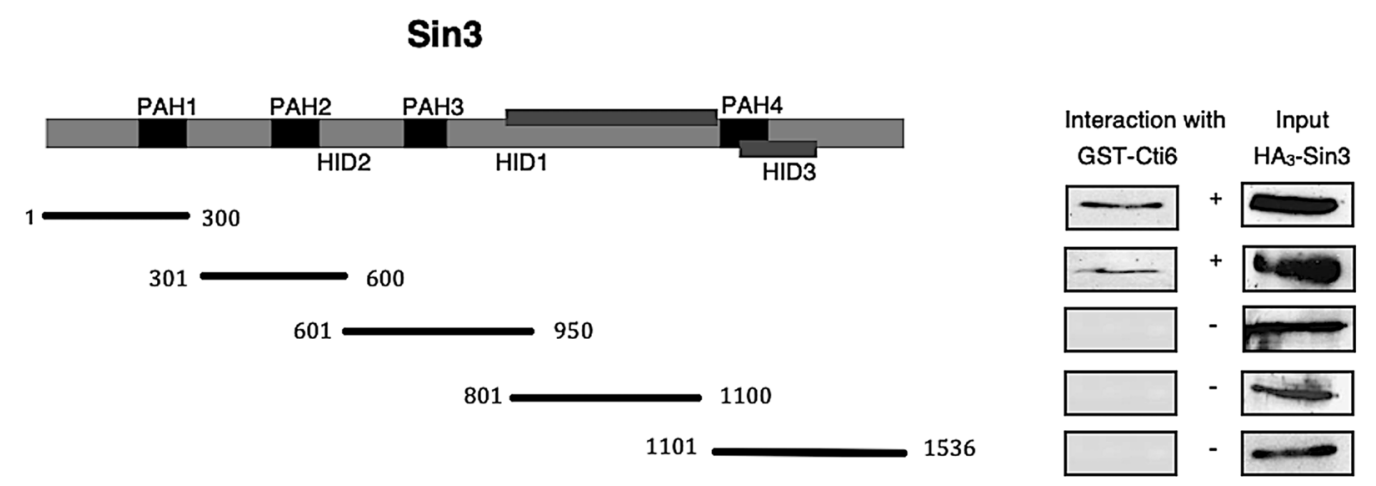

Fig. 2 Mapping of Sin3 domains interacting with Cti6. GST-Cti6 fusion plasmid pRAR3 was used to synthesize full length of Cti6. The following expression plasmids representing individual PAH domains were used for synthesis of HA-tagged Sin3 length variants in S. cerevisiae: pCW83 (aa 1-300), pYJ91 (aa 301-600), pYJ90 (aa

Gal4 activator being able to stimulate expression of the GAL2$A D E 2$ reporter gene of the recipient strain. As a negative control, empty vectors containing DBD and TAD were used.

As is shown in Fig. 3b, the negative control expectedly showed no growth on adenine-free medium. In contrast, cotransformation of DBD-Sin3 (aa 301-888) with TAD fused to Cti6 length variants restored growth on adenine-free medium (Fig. 3b), which is consistent with results from the in vitro analysis that proved interaction between $\mathrm{Cti6}$ and the PAH2 of Sin3. Importantly, Cti6 $6_{450-506}$ minimal interaction domain which has displayed in vitro interaction with PAH2 of Sin3 is able to show in vivo binding as well.

In contrast to in vitro results, DBD fusions of $\operatorname{Sin} 3$ (aa 1-300, containing PAH1) in combination with TAD fusions of Cti6 were unable to mediate growth on medium lacking adenine. Presumably, formation of functional interaction domains in vivo is prevented with certain length variants due to failure of correct protein folding. In summary, use of the "two-hybrid" system confirmed in vivo interaction between Cti6 and PAH2 of Sin3.

\section{Structural pattern within CSID}

To define the structural principles of Cti6-Sin 3 interaction, we looked for an amphipathic pattern of amino acids within a putative $\alpha$-helix of CSID. Indeed, amino acids 466-493 form such a regular pattern of hydrophobic residues (apparent from the heptad display of the Cti6 sequence shown in Fig. 4).

\section{A lexA-Cti6 fusion protein mediates transcriptional repression}

Cti6 can functionally associate in vivo with the Rpd3-Sin3 complex in S. cerevisiae (Puig et al. 2004), in addition to our
601-950), pYJ89 (aa 801-1100) and pMP20 (aa 1101-1536). For input controls (shown in the right panel of the figure), $20 \%$ of protein used for the interaction assay was analyzed. PAH1-4: paired amphipathic helices 1-4

results in this report, which definitely confirmed the direct interaction between Sin 3 and Cti6. According to these findings, the assumption that $\mathrm{Cti6}$ may act as a repressor is obvious. To test for repressor function, two strains containing integrated reporter genes (CYCl-lacZ without lexA-binding site; CYC1-lacZ with four lexA-binding sites) have been used. To quantify the repression effect of Cti6 in vivo, an effector plasmid was constructed, carrying the DNA-binding domain of the bacterial lexA repressor $\left(\operatorname{lex}_{\mathrm{DBD}}\right)$ together with the Cti6 reading frame. The empty lexA plasmid (pRTlexA) was used as a control.

If this protein actually acts as a repressor, gene activation should be reduced as a result of co-repressor recruitment (such as Sin3-HDAC), leading to inaccessible chromatin. As shown in Table 1, the tested repressor Cti6 indeed conferred a significant reduction of the reporter gene expression in the presence of lexA-binding sites. Recruitment of Cti6 to lexAbinding sites reduced the specific $\beta$-galactosidase activity more than fivefold $(9 \mathrm{U} / \mathrm{mg}$ vs. $50 \mathrm{U} / \mathrm{mg}$ in the strain without lexA-binding sites). Our results show that Cti6 mediates transcriptional repression when recruited to a promoter. This is in accordance with the findings of Puig et al. (2004) who have shown the repression function of Cti6 when targeted to promoter associated with Rpd3 HDAC.

\section{Mutational analysis of Cti6 domain interacting with Sin3}

As shown above, the C-terminus of Cti6 (residues 450-506) is able to interact with PAH2 of Sin 3 in vitro and in vivo. Within this domain (designated CSID), an amphipathic pattern of hydrophobic amino acids could be identified (residues 466-493; Fig. 4). To investigate the possible importance of these residues for interaction with Sin3 (and consequently for regulated expression of Cti6 
(a)

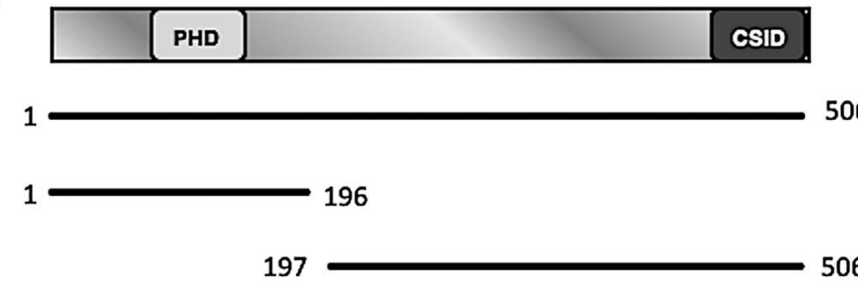

\section{Cti6}



GST vector
Interaction with $\mathrm{HA}_{3}-\mathrm{Sin} 3$


(b)

\begin{tabular}{cc}
\multicolumn{1}{c}{ DBD fusion } & TAD fusion \\
$\operatorname{Sin} 3(1-300)$ & Cti6 $(351-506)$ \\
$\operatorname{Sin} 3(1-300)$ & Cti6 $(430-506)$ \\
$\operatorname{Sin} 3(1-300)$ & Cti6 $(450-506)$ \\
$\operatorname{Sin} 3(301-888)$ & Cti6 $(351-506)$ \\
$\operatorname{Sin} 3(301-888)$ & Cti6 (430-506) \\
$\operatorname{Sin} 3(301-888)$ & Cti6 (450-506) \\
DBD & TAD
\end{tabular}


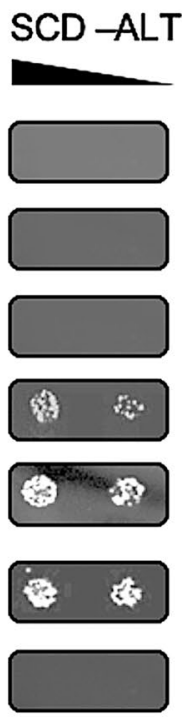

Gal4 DNA-binding domain (DBD) fused with Sin3 domains PAH1 and PAH2, respectively, were transformed into strain PJ69-4A, containing a GAL2-ADE2 fusion (selection marker: TRP1). Correspondingly, various plasmids encoding fusions of Gal4 transcriptionalactivation domain (TAD) with Cti6 were co-transformed (selection marker: LEU2): pRAR20 (Cti6, aa 351-506), pRAR37 (Cti6, aa 430-506), pRAR49 (Cti6, aa 450-506). As a negative control, empty pGBD-C1 and pGAD-C1 vectors were used. Growth in the absence of adenine is possible when a functional Gal4 activator is reconstituted by Cti6-Sin3 interaction in vivo. Selection plates (SCD-LT, absence of leucine and tryptophan; SCD-ALT, absence of leucine, tryptophan and adenine) were incubated for $48 \mathrm{~h}$ 
(466)

\begin{tabular}{|c|c|c|c|c|c|}
\hline a & b & c & d & e & $\mathbf{f}$ \\
\hline $\mathrm{F}$ & $\bar{V}$ & $\mathrm{E}$ & K & V & $\mathrm{D}$ \\
\hline I & Y & $\mathrm{N}$ & $\mathrm{G}$ & Y & $\mathrm{N}$ \\
\hline $\mathrm{S}$ & $\mathrm{L}$ & S & $\mathrm{M}$ & $\mathrm{M}$ & $\mathrm{D}$ \\
\hline $\mathrm{L}$ & $\mathrm{T}$ & $\mathrm{R}$ & $\mathrm{E}$ & $\mathrm{L}$ & $\mathrm{L}$ \\
\hline
\end{tabular}

Fig. 4 Amphipathic pattern of hydrophobic amino acids within CSID. Amino acids 466-493 of Cti6 are displayed as a heptad repeat (a-g). Hydrophobic residues at positions $\mathbf{a}, \mathbf{b}$ and $\mathbf{e}$ are boxed

Table 1 Influence of Cti6 repressor fusion plasmid on the expression of the lex $\mathrm{Ap}_{\mathrm{Op}}$-containing reporter gene

\begin{tabular}{llll}
\hline Plasmids & $\begin{array}{l}\text { Specific } \beta \text {-galactosidase activity }(\mathrm{U} / \\
\mathrm{mg})\end{array}$ & $\begin{array}{l}\text { Repres- } \\
\text { sion- } \\
\text { factor }\end{array}$ \\
\cline { 2 - 3 } & $\begin{array}{l}\text { NKTS } \\
(0 \times \text { lexA })\end{array}$ & $\begin{array}{l}\text { RTS } \\
(4 \times \text { lexA })\end{array}$ & \\
\hline pRAR27 & $\mathbf{5 0}(8)$ & $\mathbf{9}(2)$ & 5.6 \\
pRT & $\mathbf{5 1}(14)$ & $\mathbf{4 7}(19)$ & 1 \\
\hline
\end{tabular}

Both $S$. cerevisiae reporter strains NKTS (without LexA binding sites) and RTS + lexA (4 LexA binding sites) were individually transformed with pRAR27 (LexA-Cti6) and pRT (empty LexA vector) and grown in SCD-Ura-Leu liquid medium to mid- log growth phase. After cell harvesting, the specific $\beta$-galactosidase activity was determined in crude extracts of the transformants. Specific $\beta$-galactosidase activities are given in nmol ONPG hydrolyzed per min per mg of protein $(\mathrm{U} / \mathrm{mg}$ ). Each experiment represents the mean value (in bold) of 5 independent strain cultivations and enzyme assays. $\pm S D$ standard deviation. The respective standard deviation is given in parenthesis

target genes), we performed a site-directed mutagenesis at selected positions leading to replacement of hydrophobic amino acids to alanine (single mutations V467A and L481A, triple mutation L491A L492A L493A). The influence of alanine substitutions in domains of Cti6 (aa 450-506) on in vitro interaction with $\operatorname{Sin} 3$ was examined by affinity chromatography ("GST pull-down").

Expression plasmids encoding GST fusions of mutagenized Cti6 were synthesized in E. coli, bound to GSHSepharose and incubated with a protein extract from $S$. cerevisiae containing $\mathrm{HA}_{3}-\mathrm{Sin} 3_{301-600}$. GST-Cti6 ${ }_{450-506}$ representing the minimal wild-type interaction domain was used as a positive control; GST without fusion protein was used as a negative control. As shown in Fig. 5, $\mathrm{Cti6}_{450-506}$ variant V467A is still able to mediate interaction with $\mathrm{PAH} 2$, although a weakened interaction signal was obtained. In contrast, the other two mutational variants (Cti6 ${ }_{450-506}$ L481A and $\mathrm{Cti6}_{450-506}$ L491A L492A L493A) were completely defective for interaction with $\mathrm{PAH} 2$ of Sin 3 .

It can be summarized that leucine residues at amino acid positions 481, 491, 492 and 493 in CSID have a pivotal role in interaction with Sin3. However, it cannot be excluded that a single residue among L491, L492 and L493 is more

\begin{tabular}{|c|c|c|c|c|}
\cline { 2 - 5 } \multicolumn{1}{c|}{} & \multicolumn{4}{c|}{ GST-Cti6 } \\
\cline { 2 - 5 } \multicolumn{1}{c|}{} & Wild Type & V467A & L481A & $\begin{array}{r}\text { L491A, } \\
\text { L492A, } \\
\text { L493 A }\end{array}$ \\
\hline $\begin{array}{c}\text { Interaction with } \\
\mathrm{HA}_{3} \text {-Sin3 }\end{array}$ & & & & \\
\hline
\end{tabular}

Fig. 5 In vitro interaction of GST-Cti6 mutant variants and $\mathrm{HA}_{3}-\mathrm{Sin} 3$ (PAH2). GST-Cti6 ${ }_{450-506}$ comprising CSID wild type and missense variants (plasmids pRAR47, pRAR50, pRAR51 and pRAR52) were comparatively analyzed for interaction with HA-tagged $\operatorname{Sin} 3_{301-600}$ (PAH2) expressed in S. cerevisiae (plasmid pYJ91)

important for interaction than the remaining leucine residues since all amino acids were replaced simultaneously.

To assay for interaction of Cti6 wild type and missense variants with $\operatorname{Sin} 3$ in vivo, we fused Cti6 residues 450-506 with the Gal4 activation domain. The resulting plasmids were co-transformed into strain PJ69-4A with a plasmid encoding Sin 3 residues 301-888 (comprising PAH2 and PAH3) fused behind DNA-binding domain of Gal4. In the case of Cti6-Sin 3 interaction, the resulting hybrid constructs should be able to activate the Gal4-dependent GAL2-ADE2 reporter gene which restores growth on adenine-free medium when a functional Gal4 is reconstituted by interaction of the fused proteins. In contrast to the wild-type minimal interaction domain $\left(\mathrm{Cti6}_{450-506}\right)$, Cti6 variants V467A, L481A and L491A L492A L493A were unable to activate the reporter gene (Fig. 6), confirming that these Cti6 variants indeed fail to bind to PAH2 of Sin3.

\section{The Cyc8-Tup1 co-repressor targets Cti6 protein}

It has been previously reported that Cti6 directly interacts in vivo and in vitro with the co-repressor Cyc 8 (Conlan and Tzamarias 2001; Papamichos-Chronakis et al. 2002). This finding could be confirmed by our studies, again using GST pull-down assays. GST-Cti6 immobilized on GSH Sepharose could associate in vitro with recombinant HA-tagged Cyc8 synthesized in $E$. coli in the absence of additional yeast proteins (Fig. 7a). Furthermore, we also tested for a direct interaction between Cti6 and Tup1. GST-Cti6 full-length fusion was incubated with $E$. coli extract (strain BL21) containing $\mathrm{HA}_{3}$-tagged proteins Tup1 protein. As can be seen in Fig. 7b, Cti6 could indeed directly bind also to Tup1.

\section{CSID interacts with TPR motifs of Cyc8}

Since previous studies have shown binding of Sin 3 and Cyc8 to the same domain of the Opil repressor (OSID; Jäschke et al. 2011; Wagner et al. 2001), it is likely (although not inevitable) that pleiotropic co-repressors contact a common interaction domain also in other repressors. To confirm the assumption of $\mathrm{Cyc} 8$ being important 


\author{
DBD fusion \\ TAD fusion \\ Sin3 (301-888) Cti6450-506 \\ Sin3 (301-888) Cti6450-506 V467A \\ $\operatorname{Sin} 3(301-888) \quad$ Cti6450-506 L481A \\ $\operatorname{Sin} 3(301-888)$ \\ Cti6450-506 L491,492,493A \\ DBD \\ TAD
}



fusion plasmids (selection markers: TRP1 and LEU2, respectively) were co-transformed into strain PJ69-4A, containing a GAL2-ADE2 fusion that allows growth in the absence of adenine when a functional Gal4 activator is reconstituted. Selection plates (-L -T and -L -T -A; absence of leucine, tryptophan and adenine) were incubated for $48 \mathrm{~h}$. Sequence of the mutagenized Cti6 domain (residues 466-493): FVEKVDTIYNGYNESLSMMDDLTRELLLW. Amino acids that were replaced by alanine are underlined

interaction domain (CSID; aa 450-506) was also able to interact with the TPR motifs of Cyc8.

\section{$\operatorname{Sin} 3$ is recruited to iron-responsive elements (IRE) containing promoters by interacting directly with Cti6}

The functional relationship between Cti6 and Sin3 corepressor led us to the hypothesis that $\operatorname{Sin} 3$ might be present at Cti6 target genes. Chromatin immunoprecipitation assay (ChIP) was employed to monitor directly the occupancy of particular promoters by both regulatory proteins. A growing body of data points to the significant role of Cti6 in iron metabolism (Puig et al. 2004; Crisp et al. 2006). Therefore, two structural genes (SMF3 and RNR3) have been selected as targets for Cti6 regulation. $S M F 3$ is a member of the Nramp family of divalent metal transporter (Portnoy et al. 2002), which transports iron from the vacuole under ironlimited conditions (Philpott and Protchenko 2008; Kaplan and Kaplan 2009). RNR3 catalyzes the rate-limiting step in the synthesis of deoxyribonucleotides, playing a major irondependent role in DNA replication and repair (Stubbe 2003).

Accordingly, we have first examined the existence of Cti6 at promoters of both target genes under repressive conditions $(+100 \mu \mathrm{M} \mathrm{Fe}+)$. This is achieved by constructing a strain (RAY1) encoding an epitope-tagged Cti6-HA variant which was subsequently investigated by ChIP analysis. A strain encoding an epitope-tagged variant of Sin3 (Sin3-HA; FKH11) was kindly provided by F. Kliewe (Kliewe et al. 


\section{Cti6}

\section{Interaction with Cyc81-398}

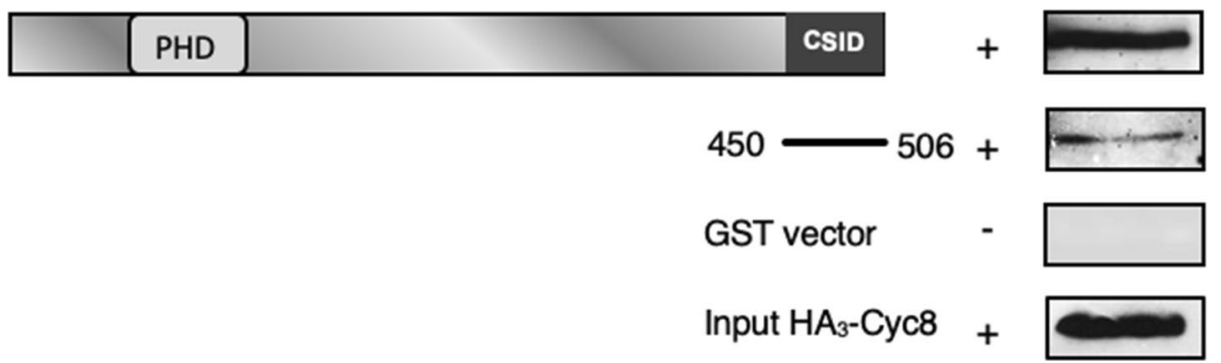

Fig. 8 In vitro interaction between length variant GST-Cti6 ${ }_{450-506}$ and TPR domains of Cyc8. Length variant GST-Cti6 ${ }_{450-506}$ was immobilized on GSH Sepharose and incubated with protein extract from E. coli (Strain BL21), containing $\mathrm{HA}_{3}-\mathrm{Cyc} 8$ (plasmid pFK77;

(a) Recruitment of Cti6 and $\operatorname{Sin} 3$ to $R N R 3$ and $S M F 3$ promoters

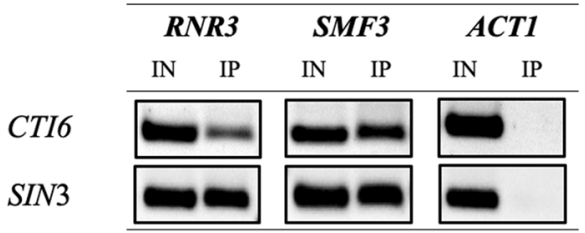

(b) Loss of $\operatorname{Sin} 3$ recruitment in the absence of Cti6

\begin{tabular}{|c|c|c|c|c|c|c|}
\hline & \multicolumn{2}{|c|}{ RNR3 } & \multicolumn{2}{|c|}{ SMF3 } & \multicolumn{2}{|c|}{$A C T 1$} \\
\hline & IN & IP & IN & IP & IN & IP \\
\hline N3 $\triangle$ cti6 & & & & & & \\
\hline
\end{tabular}

Fig. 9 Sin3 recruitment to IRE-containing promoters. Cti6-dependent recruitment of Sin3 co-repressor to IRE-containing promoter regions of $R N R 3$ and $S M F 3$ shown by chromatin immunoprecipitation. Strains RAY1 (contains a His-tagged variant of CTI6 at its natural chromosomal position), RAY3 (isogenic cti6 deletion mutant) and FKY11 (contains a His-tagged variant of $\operatorname{Sin} 3$ at its natural chromosomal position) were cultivated to the exponential growth phase under repression $(+100 \mu \mathrm{M} \mathrm{Fe}+)$. After shearing of chromatin, binding to His-Tag Dynabeads ${ }^{\circledR}$ and elution, promoter fragments were analyzed by end-point PCR (a) recruitment of Cti6 and $\operatorname{Sin} 3$ to RNR3 and $S M F 3$ promoters (b) loss of $\operatorname{Sin} 3$ recruitment in the absence of Cti6, using specific primers for RNR3, SMF3 and ACT1 (negative control). PCR products were obtained after 29 amplification cycles and then separated by electrophoresis on a $2 \%$ agarose gel. IN input control of total chromatin fragments, IP analysis of samples obtained by affinity purification

2020). As can be clearly seen in Fig. 9a, both HA-tagged Cti6 and Sin 3 proteins were tethered at $S M F 3$ and $R N R 3$ promoters under repression in wild-type cells, implicating a role of both regulators for expression of these genes. In vitro
10 TPR motifs). GST-Cti6 fusions are encoded by plasmids pRAR3 (aa 1-506) and pRAR47 (aa 450-506), respectively. GST vector was used as a negative control. Input control is shown at the bottom of the figure ( $20 \%$ of protein used for the interaction assay)

experiments have shown Sin 3 recruitment by Cti6. Therefore, we further tested whether $\operatorname{Sin} 3$ recruitment depends on the function of the Cti6 protein. A CTI6 gene deletion was introduced into strain FKH11 which encodes epitopetagged Sin3 (RAY3; Sin3-HA $\Delta c t i 6$ ) and then Sin3 recruitment to target genes was investigated again. As is apparent from Fig. 9b, Sin 3 failed to bind to both promoters in the $\Delta c t i 6$ mutant strain although Sin 3 was detected in the presence of an intact copy of Cti6. Thus, Sin 3 co-repressor can efficiently be recruited to gene promoters regulated by iron when the Cti6 protein is present.

\section{Discussion}

Cti6 was first identified as Cyc8-Tup1 interacting factor (Papamichos-Chronakis et al. 2000; Conlan and Tzamarias 2001). Additionally, it is known as a subunit of the Rpd3L histone deacetylase complex (Carrozza et al. 2005) responsible for the deacetylation of lysine residues at the N-terminal part of core histones. Histone deacetylation gives a tag for epigenetic repression and plays an important role in transcriptional regulation. Two independent reports have described the association of Cti6 with both Sin3 (Puig et al. 2004) and Cyc8/Tup1 (Papamichos-Chronakis et al. 2002).

But yet no reports investigated more specifically which domain in Cti6 is required for recruitment of the targeted corepressors. In addition, no reports have explored the domains within Sin 3 co-repressor responsible for this interaction. To deduce the features in charge of the function and regulation of co-repressors $\operatorname{Sin} 3$, Cyc8 and Tup1 in cells, it is crucial to elucidate a physical map of interacting domains within co-repressors and Cti6 as well. Former reports proposed a functional association of Cti6 with the Rpd3-Sin3-HDAC complex (Ho et al. 2002; Puig et al. 2004) while mapping 
of its Sin3 binding site has not yet been performed. So, the findings in this work are the first one to decipher such missing features.

In this report, we have clearly confirmed that Cti6 directly interacts with the pleiotropic co-repressor Sin3, indicating that the pathway-specific protein Cti6 executes its function by recruiting the general co-repressor Sin3. Data presented here also indicate that a single domain of yeast protein Cti6 (CSID; aa 450-506) is able to interact with the PAH2 of co-repressor Sin3. No interaction has been found within the C-terminus of Sin 3 which could be attributed to the fact that the C-terminal region of $\operatorname{Sin} 3$ is associated with complex subunits, such as Sap30, Pho23, etc., which render it inaccessible to other proteins. In addition, the recruitment of HDACs is brought about by interactions with regions in which PAH3 and PAH4 are located (HDACs Rpd3, Hda1 and Hos1 all bind PAH4; Grigat et al. 2012). The "Yeast two-hybrid" system has been used to confirm the examined interactions in vivo. It could be affirmed that PAH2 of Sin3 is sufficient for mediating interaction with Cti6. This finding agrees partially with the in vitro mapping results giving evidence that PAH1 and PAH2 of Sin 3 can bind to Cti6. In contrast, two-hybrid fusion constructs containing $\mathrm{Gal}_{\mathrm{TAD}} \mathrm{Cti6}_{450-506}$ (Cti6-Sin3 interaction domain, CSID) and Gal4 ${ }_{\mathrm{DBD}^{-}} \mathrm{Sin}_{1-300}$ (PAH1) could not activate the GAL2$A D E 2$ reporter gene which can be attributed to problems with protein folding of $\operatorname{Sin} 3$ partial domains under in vivo conditions.

Site-directed mutagenesis of selected hydrophobic residues (V467A, L481A and L491A L492A L493A) has been used to perform a mutational analysis of CSID. While Cti6 interaction with Sin3 appeared to be still functional with a mutated valine (V467A) in vitro (GST pull-down assay), all tested Cti6 variants were defective for the interaction with Sin 3 in vivo (two-hybrid assay), indicating that these positions are indispensable for Cti6 binding to Sin3. We suggest that variant V467A may show some residual function which allows interaction in vitro as a result of increased protein amount but is not sufficiently functional in vivo. Although the plant homeodomain (PHD) of Cti6 has an important role for GAL1 transcriptional activation (Papamichos-Chronakis et al. 2002) and for growth under conditions of iron scarcity as well, our mapping studies demonstrated that this domain is dispensable for its interaction with $\operatorname{Sin} 3$.

To obtain further insights into CSID features, we have tested its ability to recruit Cyc8 as well. Interestingly, CSID not only binds to PAH2 of Sin3 but also interacts with TPR motifs of co-repressor Cyc8. Cti6 has been shown to participate in the interaction of the Cyc8-Tup1 co-repressor with the Gcn5-containing SAGA histone acetyltransferase complex (Puig et al. 2002). Moreover, it has been earlier described that amino acid residues 409-506 of Cti6 mediate the interaction with Cyc8 (Papamichos-Chronakis et al.
2002). In this work, we could show that the even shorter CSID (residues 450-506) is able to mediate the interaction with $\mathrm{Cyc}_{1-398}$ (containing only the TPR motifs). In summary, we provide evidence that CSID may be involved in a second pathway of repression acting in parallel to Sin3. Interaction of two co-repressor complexes with a single specific repressor may be the exception but not a general rule, at least in yeast. Besides Cti6, previous reports substantiated that yeast repressor Opil being responsible for repression of phospholipid biosynthetic genes contacts Sin 3 and Cyc8/Tup1 via a single domain (Jäschke et al. 2011; Wagner et al. 2001). Binding of CSID to PAH and TPR motifs suggests that both interaction modules should be able to contact related targets. Moreover, we also proved the direct interaction of Cti6 with Tup1.

In this work, a versatile in vivo repressor test system was established which allows to confirm a protein as a transcriptional repressor, using a variant strategy initially described by Kadosh and Struhl (1997). We could confirm that Cti6 is able to repress transcription when fused to the DNA-binding domain of LexA. As described by Kadosh and Struhl (1997), amino acids $508-836$ of repressor Ume 6 contain the Sin 3 interaction domain which is sufficient to cause gene repression (decrease of gene expression to approx. 17\%) but only in the presence of a functional $\operatorname{Sin} 3$ co-repressor.

Thus, expression of truncated variants of lexA-repressor fusion proteins in a suitable reporter strain can be exploited for mapping of repression domains. In this report we showed that Cti6 fused to lexA could reduce the expression of the lexA-dependent reporter gene as efficient as Ume6 (decrease of gene expression to approx. 14\%). We hypothesize that Cti6 mediates this repression through recruitment of corepressors Sin3 and Cyc8-Tup1. In future work, construction of SIN3 and CYC8 gene deletions mutants could provide evidence for the relative importance of these co-repressors for reduction of gene expression. It is expected that the repression effect is abolished when co-repressors are absent. Previously, Puig et al. (2004) demonstrated that Cti6-mediated transcriptional repression was substantially alleviated in the absence of the histone deacetylase Rpd3.

To provide further mechanistic insight on the function of Cti6 and its association with $\operatorname{Sin} 3$ co-repressor, it is crucial to elucidate the role of proteins that function with this pleiotropic co-repressor, such as Cti6. Two structural genes (RNR3 and SMF3) have been selected as targets for Cti6 regulation. Both structural genes are playing an important role in iron metabolism (Puig et al. 2004; Stubbe 2003). In this work, a functional association of Cti6 with Sin 3 co-repressor could be proved by chromatin immunoprecipitation. The in vivo occupancy of $R N R 3$ and $S M F 3$ promoters by $\mathrm{Cti} 6$ and $\mathrm{Sin} 3$ has been individually monitored under repression. Furthermore, we assayed the occupancy of $\operatorname{Sin} 3$ on the respective promoters 




Fig. 10 A schematic representation of two hypothetical models of Sin 3 dependent Cti6 recruitment. RNR3 and SMF3 are iron-regulated structural genes. Under repression $(+100 \mu \mathrm{M} \mathrm{Fe}+)$, Anchoring of Cti6 on the DRE region of RNR3 and AFT of SMF3 promoters occurs. This may be implemented via Cti6 recruitment by Rfx 1 and Aft2 (in dashed oval shape as speculative scenario), respectively. Then, Cti6 recruits Sin3 co-repressor through the interaction between $\mathrm{PAH} 2$ and CSID which triggers a conformational reorganization,

by constructing a strain lacking Cti6. Results of our ChIP analysis confirmed that $\operatorname{Sin} 3$ is no longer present at these promoters in the absence of $\mathrm{Cti6}$ ( $\Delta$ cti6 mutant), indicating that $\operatorname{Sin} 3$ recruitment depends on Cti6. These results are in agreement with data of Puig et al. (2004) who demonstrated that $S M F 3$ and $R N R 3$ were up-regulated in a cti6 mutant. This allows the assumption that both pleiotropic co-repressors Sin3 and Cyc8/Tup1 work together via the interaction with Cti6 leading to gene repression.

Cti6 is recruited to iron-responsive promoters in an Aft1-dependent manner and mediates the expression of ARN1 under heme-deficient conditions (Crisp et al. 2006). As Cti6 is not a DNA-binding protein, the question arises how $\mathrm{Cti} 6$ tethers to the structural gene promoters and mediates $\operatorname{Sin} 3$ recruiting. We propose two different models concerning each structural gene. Ribonucleotide-diphosphate reductase (encoded by $R N R 3$ ) catalyzes the rate-limiting step in deoxyribonucleotide synthesis and plays an essential iron-dependent role in DNA replication and repair (Stubbe 2003). RNR3 repression is mediated by upstream repression sequences (URS) designated as damage-responsive elements (DREs) that serve as binding sites for the sequence-specific DNA-binding protein Rfx1 (Huang et al. 1998).

Accordingly, it could be suggested that under repressing conditions Cti6 binding to the $R N R 3$ promoter occurs in this order, Rfx 1 binds to the structural gene promoter, allowing entry of Cti6 which mediates repression through Sin3 and Cyc8/Tup1 recruitment. This assumption is strongly supported by the findings of Bing and Joseph (2001) who could show that $R N R 3$ repression is mediated by Cyc 8 , Tup 1 and $\mathrm{Rfx} 1$, causing changes in nucleosome positioning and chromatin structure. Therefore, the supposed interaction between Cti6 and Rfx 1 should be investigated in the future. It should be emphasized that

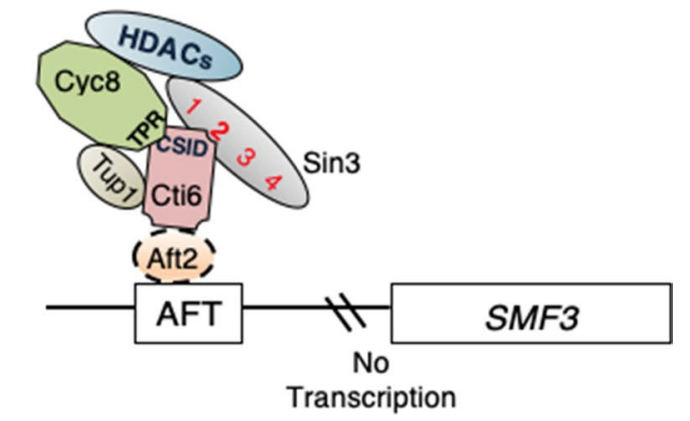

bringing HDACs into action preventing transcription of the respective genes. The existence of Cyc 8 and Tup 1 should be considered through CSID/TPR and Cti6/Tup1 interaction. DRE, damage-responsive elements; AFT, activator of Fe transcription; CSID, Cti6-Sin3 interaction domain; TPR, tetratricopeptide repeat. Numbers 1, 2, 3 and 4 within the Sin3 oval indicate paired amphipathic helices PAH1, 2, 3 and 4

direct in vitro interaction between $\mathrm{Rf} x 1$ and $\mathrm{Cyc} 8$ has been confirmed in independent work (Huang et al.1998; Zhang and Reese 2005).

A related, but slightly different situation may be effective in the case of $S M F 3$ whose upstream region possesses a pair of Aft consensus binding sequences (Portnoy et al. 2002). The DNA-binding protein Aft 2 (Blaiseau et al. 2001; Rutherford et al. 2003) binds and activates the transcription of SMF3 (Courel et al. 2005). Interestingly, $S M F 3$ is not only induced under conditions of iron starvation (Portnoy et al. 2000), but also up-regulated in cti6 mutant (Puig et al. 2004). Thence, as Cti6 is not a DNAbinding protein and can be detected on $S M F 3$ gene promoter by ChIP assay, we assume that $\mathrm{Cti} 6$ was recruited by Aft2 under repression $(+100 \mu \mathrm{M} \mathrm{Fe}+)$ inhibiting $S M F 3$ expression by recruiting the pleiotropic co-repressors $\operatorname{Sin} 3$ and Cyc8/Tup1. Further work should investigate the possible direct or indirect interaction between $\mathrm{Cti} 6$ and $\mathrm{Aft} 2$ under both repression and de-repression conditions. Both hypothetical models are summarized in Fig. 10. Taken together, our results unraveled novel structural insights into the multifunctional regulator Cti6 as we could precisely identify the critically important domain within Cti6 that directly recruits not only Sin3 (via PAH2 domain) but also Cyc8 (via TPR motifs) co-repressors. In addition, our findings provide unprecedented evidence of $\operatorname{Sin} 3$ in vivo binding on promoters of genes involved in iron response only in the presence of Cti6. In conclusion, this work has addressed the functional role of member of Rpd3L histone deacetylase complex in yeast model supporting the evidence that one domain (CSID) is implicated with multiple co-repressor complexes Sin3 and Cyc8-Tup1 in related subtle molecular events. 
Acknowledgement This work has been supported by the German Academic Exchange Service (DAAD) and Deutsche Forschungsgemeinschaft (DFG).

Author contributions JS Principle investigator of the project, checked the manuscript and made the corrections. RA constructed the respective plasmids and strains, performed the experiments, developed methodology, analyzed data, wrote manuscript and responded to manuscript submission.

Funding Open Access funding enabled and organized by Projekt DEAL.

\section{Compliance with ethical standards}

Conflict of interest The authors have no conflict of interest to declare.

Open Access This article is licensed under a Creative Commons Attribution 4.0 International License, which permits use, sharing, adaptation, distribution and reproduction in any medium or format, as long as you give appropriate credit to the original author(s) and the source, provide a link to the Creative Commons licence, and indicate if changes were made. The images or other third party material in this article are included in the article's Creative Commons licence, unless indicated otherwise in a credit line to the material. If material is not included in the article's Creative Commons licence and your intended use is not permitted by statutory regulation or exceeds the permitted use, you will need to obtain permission directly from the copyright holder. To view a copy of this licence, visit http://creativecommons.org/licenses/by/4.0/.

\section{References}

Adams GE, Chandru A, Cowley SM (2018) Co-repressor, Co-activator and general transcription factor: the many faces of the Sin3 Histone Deacetylase (HDAC) Complex. Biochem J 475:3921-3932. https://doi.org/10.1042/BCJ20170314

Bing L, Joseph R (2001) Ssn6-Tup1 regulates RNR3 by positioning nucleosomes and affecting the chromatin structure at the upstream repression sequence. J Biol Chem 276:33788-33797. https://doi. org/10.1074/jbc.M104220200

Blaiseau PL, Lesuisse E, Camadro JM (2001) Aft2p, a novel iron-regulated transcription activator that modulates, with Aftlp, intracellular iron use and resistance to oxidative stress in yeast. J Biol Chem 276:34221-34226. https://doi.org/10.1074/jbc.M104987200

Cairns BR (2009) The logic of chromatin architecture and remodelling at promoters. Nature 461:193-198. https://doi.org/10.1038/ nature 08450

Carrozza MJ, Florens L, Swanson SK, Shia WJ, Anderson S, Yates J, Washburn MP, Workman JL (2005) Stable incorporation of sequence specific repressors Ash1 and Ume6 into the Rpd3L complex. Biochim Biophys Acta 1731:77-87. https://doi. org/10.1016/j.bbaexp.2005.09.005

Chaubal A, Pile LA (2018) Same agent, different messages: insight into transcriptional regulation by SIN3 isoforms. Epigenetics Chromatin 11:17. https://doi.org/10.1186/s13072-018-0188-y

Cobb J, van Attikum H (2010) Mapping genomic targets of DNA helicases by chromatin immunoprecipitation in Saccharomyces cerevisiae. Methods Mol Biol 587:113-126. https://doi. org/10.1007/978-1-60327-355-8_8

Conlan RS, Tzamarias D (2001) Sfl1 functions via the co-repressor Ssn6-Tup1 and the cAMP-dependent protein kinase Tpk2. J Mol Biol 309:1007-1015. https://doi.org/10.1006/jmbi.2001.4742
Courel M, Lallet S, Camadro JM, Blaiseau PL (2005) Direct activation of genes involved in intracellular iron use by the yeast iron-responsive transcription factor Aft2 without its paralog Aft1. Mol Cell Biol 25:6760-6771. https://doi.org/10.1128/ MCB.25.15.6760-6771.2005

Crisp RJ, Adkins EM, Kimmel E, Kaplan J (2006) Recruitment of Tup1p and Cti6p regulates heme-deficient expression of Aft1p target genes. EMBO J 25:512-521. https://doi.org/10.1038/sj.emboj .7600961

Davie JK, Edmondson DG, Coco CB, Dent SY (2003) Tup1-Ssn6 interacts with multiple class I histone deacetylases in vivo. J Biol Chem 278:50158-50162. https://doi.org/10.1074/jbc.M3097 53200

De Antoni A, Gallwitz D (2000) A novel multi-purpose cassette for repeated integrative epitope tagging of genes in Saccharomyces cerevisiae. Gene 246:179-185. https://doi.org/10.1016/s0378 -1119(00)00083-4

Gavin AC, Bosche M, Krause R, Grandi P, Marzioch M, Bauer A, Schultz J, Rick JM, Michon AM, Cruciat CM, Remor M, Hofert C, Schelder M, Brajenovic M, Ruffner H, Merino A, Klein K, Hudak M, Dickson D, Rudi T, Gnau V, Bauch A, Bastuck S, Huhse B, Leutwein C, Heurtier MA, Copley RR, Edelmann A, Querfurth E, Rybin V, Drewes G, Raida M, Bouwmeester T, Bork P, Seraphin B, Kuster B, Neubauer G, Superti-Furga G (2002) Functional organization of the yeast proteome by systematic analysis of protein complexes. Nature 415:141-147. https://doi.org/10.1038/415141a

Grigat M, Jäschke Y, Kliewe F, Pfeifer M, Walz S, Schüller HJ (2012) Multiple histone deacetylases are recruited by co-repressor Sin 3 and contribute to gene repression mediated by Opi 1 regulator of phospholipid biosynthesis in the yeast Saccharomyces cerevisiae. Mol Genet Genom 287:461-472. https://doi. org/10.1007/s00438-012-0692-x

Ho Y, Gruhler A, Heilbut A, Bader GD, Moore L, Adams SL, Millar A, Taylor P, Bennett K, Boutilier K, Yang L, Wolting C, Donaldson I, Schandorff S, Shewnarane J, Vo M, Taggart J, Goudreault M, Muskat B, Alfarano C, Dewar D, Lin Z, Michalickova K, Willems AR, Sassi H, Nielsen PA, Rasmussen KJ, Andersen JR, Johansen LE, Hansen LH, Jespersen H, Podtelejnikov A, Nielsen E, Crawford J, Poulsen V, Sorensen BD, Matthiesen J, Hendrickson RC, Gleeson F, Pawson T, Moran MF, Durocher D, Mann M, Hogue CW, Figeys D, Tyers M (2002) Systematic identification of protein complexes in Saccharomyces cerevisiae by mass spectrometry. Nature 415:180-183. https ://doi.org/10.1038/415180a

Huang M, Zhou Z, Elledge SJ (1998) The DNA replication and damage checkpoint pathways induce transcription by inhibition of the Crt1 repressor. Cell 94:595-605. https://doi.org/10.1016/s0092 -8674(00)81601-3

James P, Halladay J, Craig EA (1996) Genomic libraries and a host strain designed for highly efficient two-hybrid selection in yeast. Genetics 144:1425-1436

Jäschke Y, Schwarz J, Clausnitzer D, Müller C, Schüller HJ (2011) Pleiotropic co-repressors Sin3 and Ssn6 interact with repressor Opi1 and negatively regulate transcription of genes required for phospholipid biosynthesis in the yeast Saccharomyces cerevisiae. Mol Genet Genom 285:91-100. https://doi.org/10.1007/s0043 8-010-0589-5

Kadosh D, Struhl K (1997) Repression by Ume6 involves recruitment of a complex containing Sin 3 co-repressor and Rpd3 histone deacetylase to target promoters. Cell 89:365-371. https://doi. org/10.1016/s0092-8674(00)80217-2

Kadosh D, Struhl K (1998) Targeted recruitment of the Sin3-Rpd3 histone deacetylase complex generates a highly localized domain of repressed chromatin in vivo. Mol Cell Biol 18:5121-5127. https ://doi.org/10.1128/mcb.18.9.5121 
Kaplan CD, Kaplan J (2009) Iron acquisition and transcriptional regulation. Chem Rev 109:4536-4552. https://doi.org/10.1021/cr900 1676

Kaplan J, McVey DW, Crisp RJ, Philpott CC (2006) Iron-dependent metabolic remodeling in S. cerevisiae. Biochim Biophys Acta 1763:646-651. https://doi.org/10.1016/j.bbamcr.2006.03.008

Kliewe F, Engelhardt M, Aref R, Schüller HJ (2020) Correction to: Promoter recruitment of corepressors $\operatorname{Sin} 3$ and Cyc 8 by activator proteins of the yeast Saccharomyces cerevisiae. Curr Genet 63:739-750. https://doi.org/10.1007/s00294-020-01097-5

Lemon B, Tjian R (2000) Orchestrated response: a symphony of transcription. Nature 461:193-198. https://doi.org/10.1101/gad.83100 0

Maqani N, Fine RD, Shahid M, Li M, Enriquez-Hesles E, Smith JS (2018) spontaneous mutations in cyc8 and mig 1 suppress the short chronological lifespan of budding yeast lacking snf1/ampk. Microb Cell 5:233-248. https://doi.org/10.15698/mic2018.05.630

Mumberg D, Müller R, Funk M (1994) Regulatable promoters of Saccharomyces cerevisiae: comparison of transcriptional activity and their use for heterologous expression. Nucl Acids Res 22:57675768. https://doi.org/10.1093/nar/22.25.5767

Papamichos-Chronakis M, Conlan RS, Gounalaki N, Copf T, Tzamarias D (2000) Hrs 1/Med3 is a Cyc8-Tup1 corepressor target in the RNA polymerase II holoenzyme. J Biol Chem 275:83978403. https://doi.org/10.1074/jbc.275.12.8397

Papamichos-Chronakis M, Petrakis T, Ktistaki E, Topalidou I, Tzamarias D (2002) Cti6, a PHD domain protein, bridges the Cyc8-Tup1 co-repressor and the SAGA coactivator to overcome repression at GAL1. Mol Cell 9:1297-1305. https://doi. org/10.1016/s1097-2765(02)00545-2

Philpott C, Protchenko OV (2008) Response to iron deprivation in Saccharomyces cerevisiae. Eukaryot Cell 7:20-27. https://doi. org/10.1128/EC.00354-07

Portnoy ME, Liu XF, Culotta VC (2000) Saccharomyces cerevisiae expresses three functionally distinct homologues of the nramp family of metal transporters. Mol Cell Biol 20:7893-7902. https ://doi.org/10.1128/mcb.20.21.7893-7902.2000

Portnoy ME, Jensen LT, Culotta VC (2002) The distinct methods by which manganese and iron regulate the Nramp transporters in yeast. Biochem J 362:119-124. https://doi.org/10.1042/02646021:3620119

Puig S, Lee J, Lau M, Thiele DJ (2002) Biochemical and genetic analyses of yeast and human high affinity copper transporters suggest a conserved mechanism for copper uptake. J Biol Chem 277:2602126030. https://doi.org/10.1074/jbc.M202547200

Puig S, Lau M, Thiele DJ (2004) Cti6 is an Rpd3-Sin3 histone deacetylase-associated protein required for growth under iron-limiting conditions in Saccharomyces cerevisiae. J Biol Chem 279:3029830306. https://doi.org/10.1074/jbc.M313463200

Rutherford JC, Jaron S, Winge DR (2003) Aft1p and Aft2p mediate iron-responsive gene expression in yeast through related promoter elements. J Biol Chem 278:27636-27643. https://doi.org/10.1074/ jbc.M300076200

Schwank S, Ebbert R, Rautenstrauss K, Schweizer E, Schüller HJ (1995) Yeast transcriptional activator INO2 interacts as an Ino2p/ Ino4p basic helix-loop-helix heteromeric complex with the inositol/choline-responsive element necessary for expression of phospholipid biosynthetic genes in Saccharomyces cerevisiae. Nucl Acids Res 23:230-237. https://doi.org/10.1093/nar/23.2.230

Stubbe J (2003) Di-iron-tyrosyl radical ribonucleotide reductases. Curr Opin Chem Biol 7:183-188. https://doi.org/10.1016/s1367 $-5931(03) 00025-5$

Tzamarias D, Struhl K (1994) Functional dissection of the yeast Cyc8Tup1 transcriptional co-repressor complex. Nature 369:758-776. https://doi.org/10.1038/369758a0

Váchová L, Palková Z (2019) Diverse roles of Tup1p and Cyc8p transcription regulators in the development of distinct types of yeast populations. Curr Genet 65:147-151. https://doi.org/10.1007/ s00294-018-0883-z

Wagner C, Dietz M, Wittmann J, Albrecht A, Schüller HJ (2001) The negative regulator Opi1 of phospholipid biosynthesis in yeast contacts the pleiotropic repressor Sin3 and the transcriptional activator Ino2. Mol Microbiol 41:155-166. https://doi.org/10.1 046/j.1365-2958.2001.02495.x

Wang H, Stillman DJ (1993) Transcriptional repression in Saccharomyces cerevisiae by a SIN3-LexA fusion protein. Mol Cell Biol 13:1805-1814. https://doi.org/10.1128/mcb.13.3.1805

Wang H, Clark I, Nicholson PR, Herskowitz I, Stillman DJ (1990) The Saccharomyces cerevisiae SIN3 gene, a negative regulator of $\mathrm{HO}$, contains four paired amphipathic helix motifs. Mol Cell Biol 10:5927-5936. https://doi.org/10.1128/mcb.10.11.5927

Zhang Z, Reese JC (2005) Molecular genetic analysis of the yeast repressor $\mathrm{Rf} \times 1 / \mathrm{Crt} 1$ reveals a novel two-step regulatory mechanism. Mol Cell Biol 25:7399-7411. https://doi.org/10.1128/ MCB.25.17.7399-7411.2005

Publisher's Note Springer Nature remains neutral with regard to jurisdictional claims in published maps and institutional affiliations. 